\title{
Large Right Ventricular Myxoma in a 79-Year-Old Male
}

\author{
Yohkoh Soejima, Akihiro Niwa, Masato Tanaka, Mayumi Doi, Masao NitTa, Toshihiko TaKamoto*, \\ Michiaki Hiroe*, Fumiaki Marumo*, Toshizumi ShiraI**, Masazumi Watanabe** and Akio Suzuki**
}

\begin{abstract}
A 79-year-old male, admitted because of severe dyspnea on exertion, showed echocardiographic findings of a large tumor in the dilated right ventricle. The right ventricular outflow tract was nearly occluded by the tumor mass, and the mass was attached to the interventricular septum by a pedicle. The tumor removal operation was successful. The size of the tumor was $40 \mathrm{~mm} \times 90 \mathrm{~mm}$, and the weight $70 \mathrm{~g}$. Microscopic findings showed typical myxomatous tissue with high cellularity, and no malignancy was observed. This is the oldest reported patient with right ventricular myxoma which was cured by operation.
\end{abstract}

(Internal Medicine 35: 380-382, 1996)

Key words: right ventricular outflow tumor, pulmonic valvular occlusion, right heart failure

\section{Case Report}

Myxoma most typically develops in the atria, and rarely in the ventricles. Recently, we treated a rare case of large right ventricular myxoma in a 79-year-old male. He had the symptom of dyspnea on effort for several years, and was admitted to our hospital on October 26, 1990 due to worsening of that symptom. Physical findings showed regular pulsation of $130 / \mathrm{min}$, and blood pressure of $102 / 64 \mathrm{mmHg}$ in the supine position, but it was not possible to measure his blood pressure and pulsation in the sitting position. He had no lymphadenopathy or pretibial edema. Auscultation showed decreased second heart sound without murmur, normal vesicular sound with no rales. Abdomen was soft and flat without any mass palpable, or ascites. Laboratory findings showed the following: white blood cell count $6,800 / \mathrm{mm}^{3}$, hemoglobin $16.4 \mathrm{mg} / \mathrm{dl}$, hematocrit $49.3 \%$, platelet $9.3 \times 10^{4} / \mathrm{mm}^{3}$, glutamic oxalacetic transaminase $103 \mathrm{U} /$ $l$, glutamic pyruvic transaminase $123 \mathrm{U} / l$, lactic dehydrogenase $1,571 \mathrm{IU} / l$, creatinine phosphokinase $69 \mathrm{IU} / l$, creatinine 1.3 $\mathrm{mg} / \mathrm{dl}$, sodium $134 \mathrm{mEq} / l$, potassium $4.6 \mathrm{mEq} / l$, chloride 100 $\mathrm{mEq} / \mathrm{l}$. Analysis of arterial blood gas in the room air showed $\mathrm{PO}_{2}$ of 65 Torr, $\mathrm{PCO}_{2}$ of 27 Torr, and $\mathrm{HCO}_{3}$ of $19 \mathrm{mEq} / l$. On electrocardiogram, normal sinus rhythm with complete right bundle branch block was observed. There was no abnormal finding on the chest X-ray picture. On the echocardiogram, a large tumor mass was seen in the dilated right ventricle, and the left ventricle showed a small deformed cavity (Fig. 1). The right ventricular outflow tract was nearly occluded by the tumor mass. The mass was attached to the interventricular septum by a pedicle; it was mobile inside the right ventricular outflow tract, moving toward the pulmonic valve in systole (Fig. 2). On the 10th hospital day, cardiac catheterization examination was done. The central venous pressure was 21 Torr, and the right ventricular pressure 66/8 Torr (end-diastolic pressure 22 Torr). Coronary angiography revealed no abnormality. Right ventriculography revealed a large defect in the outflow tract region (Fig. 3). Because of rapidly progressing liver dysfunction due to right heart failure, an emergency operation was done on the 12th hospital day. The right ventricular outflow tract was markedly swelled without contractility by the tumor mass, and the right atrium was dilated and hypertrophied, suggestive of pressure overload. After making an incision in the right atrium, a large tumor mass was seen along the right ventricular outflow tract, attached to the interventricular septum by a pedicle. The tumor was resected by carrying out an incision into the pulmonary artery trunk. The size of the tumor was $40 \mathrm{~mm}$ (diameter) $\times 90 \mathrm{~mm}$ (length), and the weight was $70 \mathrm{~g}$ (Fig. 4). Microscopic findings showed typical myxomatous tissue with high cellularity, and no malignancy was observed (Fig. 5). Since then, he has been followed on an outpatient basis in stable condition, without right heart failure or liver dysfunction.

\section{Discussion}

Primary cardiac tumor is relatively rare, and the proportion of the myxoma occupies more than half of the primary tumor

From the Cardiovascular Division, Musashino Red Cross Hospital, Tokyo, *the Second Department of Medicine and **the Department of Cardiovascular \& Thoracic Surgery, Tokyo Medical and Dental University, Tokyo 

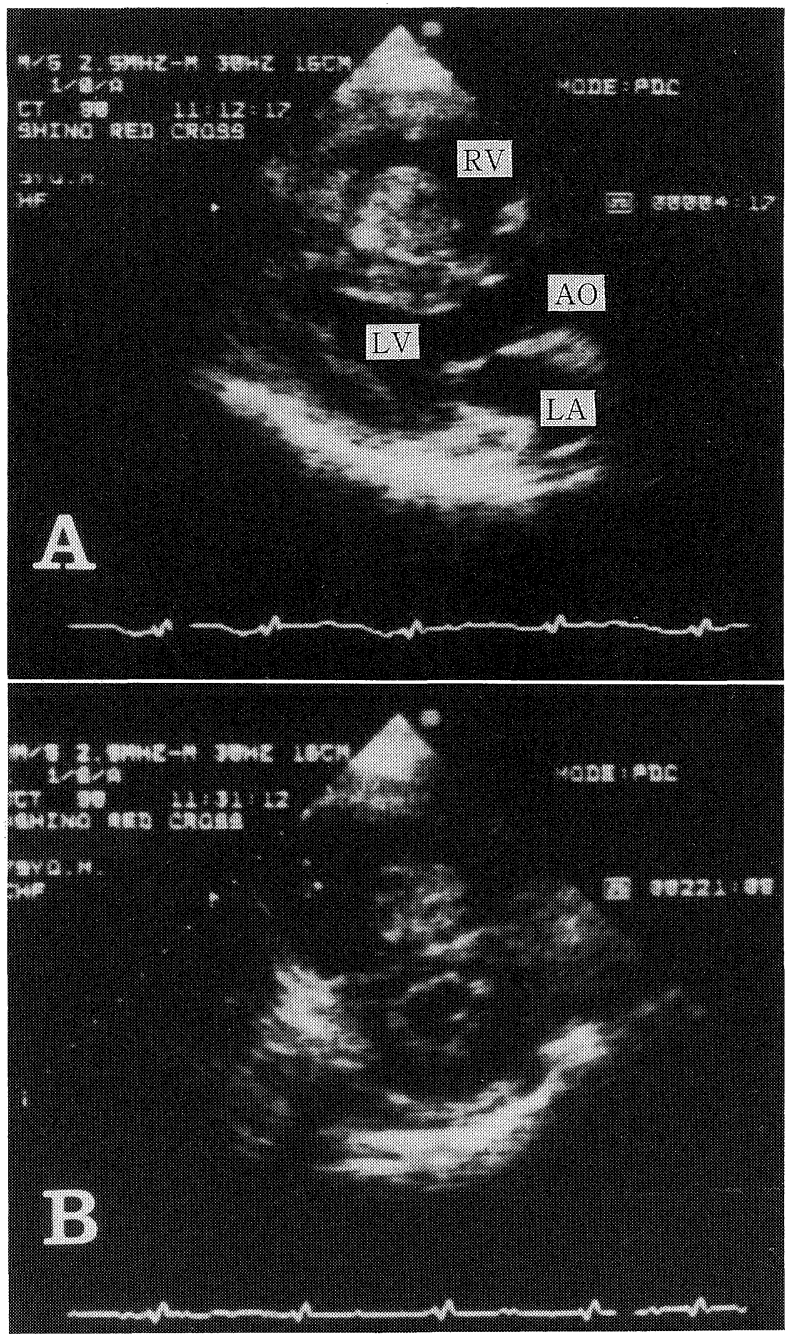

Figure 1. Transthoracic echocardiogram. A large tumor mass was seen in the dilated right ventricle, and the left ventricle showed a small deformed cavity. A) long axis view, B) short axis view. AO: ascending aorta, LA: left atrium, LV: left ventricle, $R V$ : right ventricle.

(1). Cardiac myxoma always originates in the atria, and rarely in the ventricles (1-4). The typical clinical symptom of right ventricular myxoma is illustrated as dyspnea, chest pain, syncopal attack, and cyanosis (1,3-5). This inability to measure the blood pressure in the sitting position may reflect the pulmonic valvular occlusion by the tumor mass $(3,5,6)$. The present patient is the oldest reported case of right ventricular myxoma cured by operation $(4,5,7)$. The tumor had grown gradually for a long time to nearly obstruct the right ventricular outflow tract (6), and was successfully and safely resected by operation. Therefore, it is important that resection of the right ventricular myxoma is achieved as quickly as possible even in an old patient such as this case, to prevent serious complications that can ensue in untreated patients $(5,8)$.

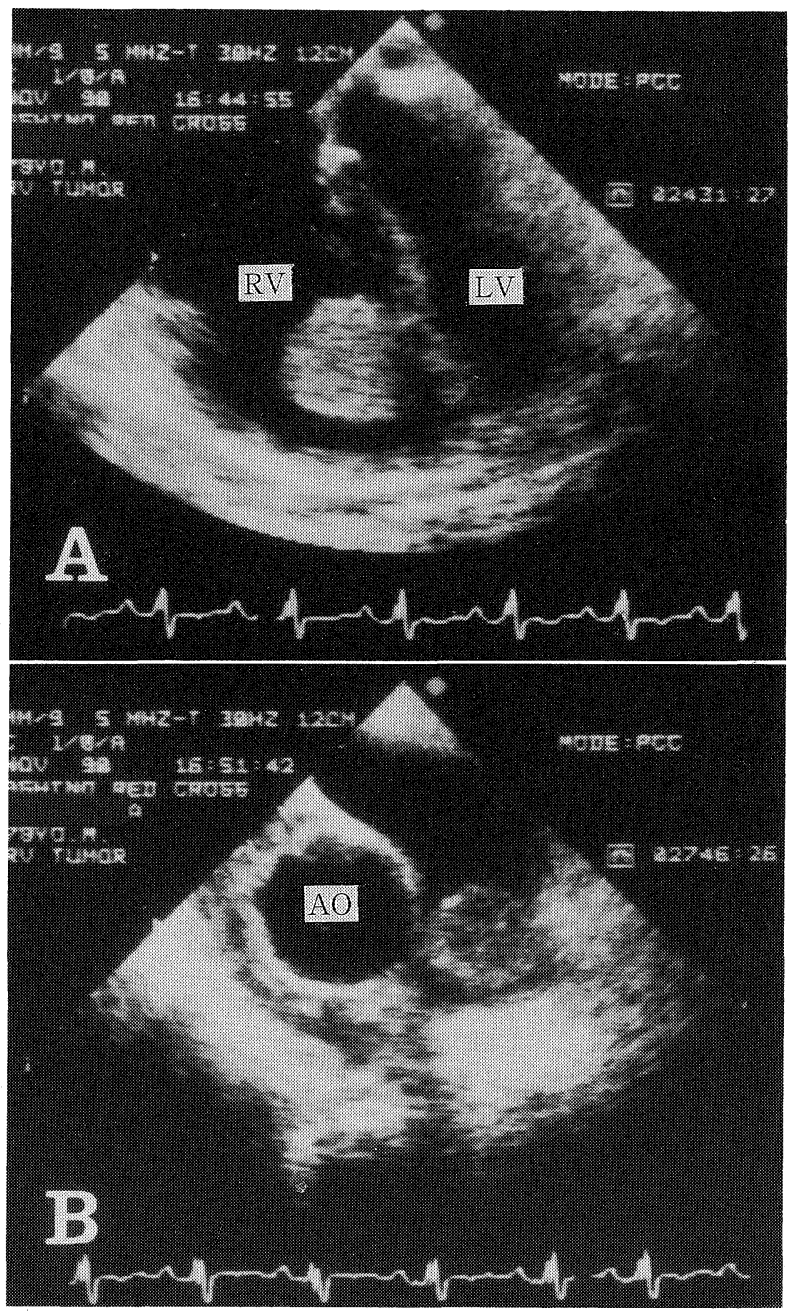

Figure 2. Transesophageal echocardiogram. Right ventricular outflow tract was nearly occluded by the tumor mass (B), and the mass was attached to the interventricular septum by a pedicle (A). AO: ascending aorta, $L V$ : left ventricle, $R V$ : right ventricle.

\section{References}

1) González A, Altieri PI, Màrquez EU, Cox RA, Castillo M. Massive pulmonary embolism associated with a right ventricular myxoma. Am J Med 69: 795, 1980.

2) Ports TA, Schiller NB, Strunk BL. Echocardiography of right ventricular tumors. Circulation 56: 439, 1977.

3) Chandraratna PAN, San Pedro S, Elkins RC, Grantham N. Echocardiographic, angiocardiographic, and surgical correlations in right ventricular myxoma simulating valvar pulmonic stenosis. Circulation 55: $619,1977$.

4) Bortolotti U, Mazzucco A, Valfré C, Valente M, Pennelli N, Gallucci V. Right ventricular myxoma: Review of the literature and report of two patients. Ann Thorac Surg 33: 277, 1982.

5) Snyder SN, Smith DC, Lau FYK, Turner AF. Diagnostic features of right ventricular myxoma. Am Heart J 91: 240, 1976. 


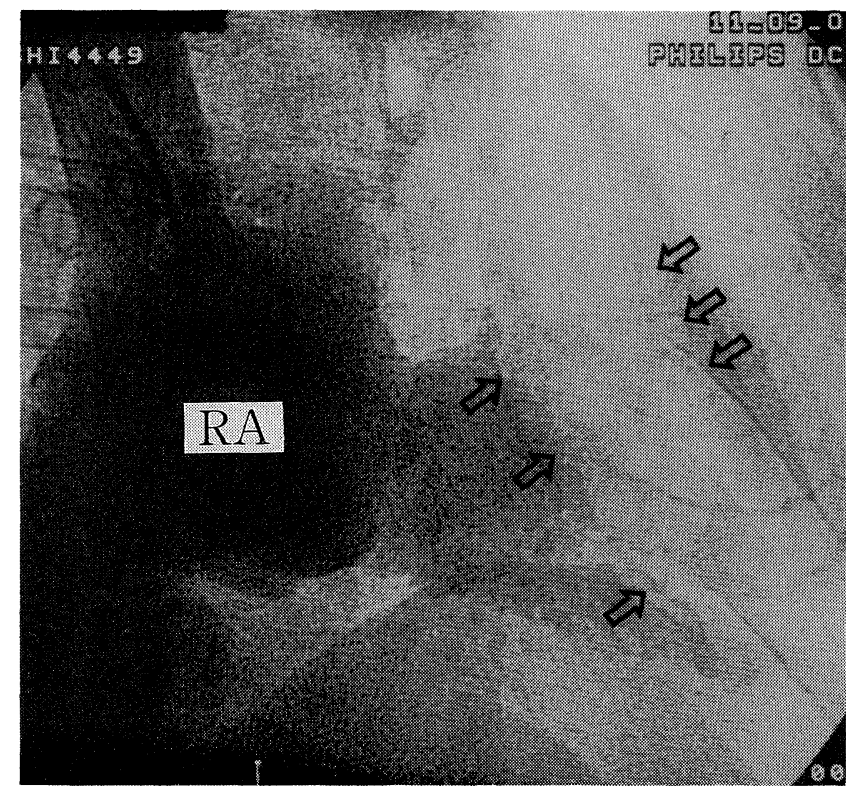

Figure 3. Right ventriculography with contrast medium injected at the superior vena cava revealed a large defect in the outflow tract region (arrows). RA: right atrium.

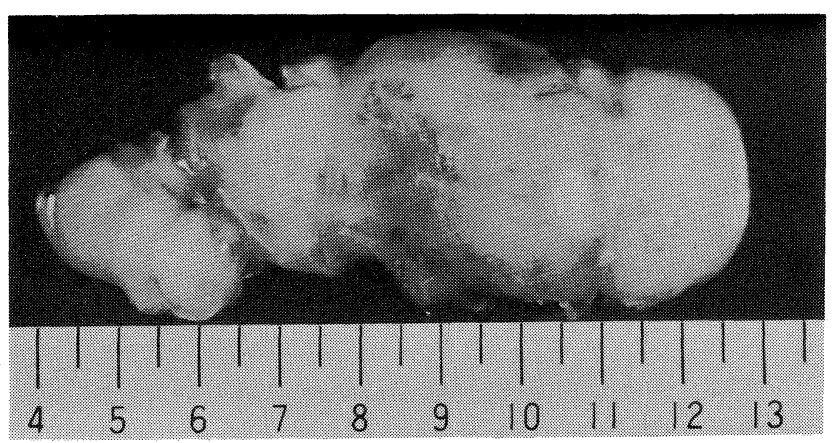

Figure 4. Macroscopic findings of the right ventricular myxoma.

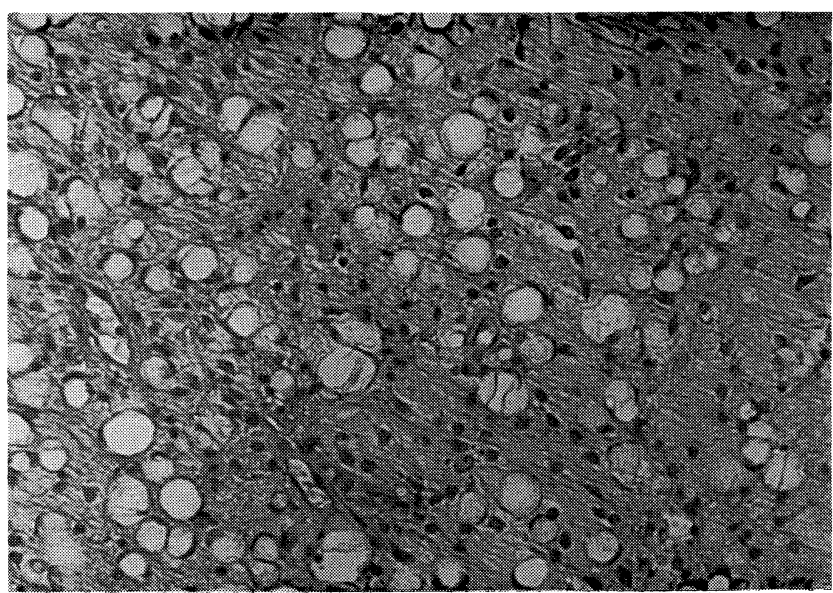

Figure 5. Microscopic findings of the right ventricular myxoma (HE, $\times \mathbf{4 0 0})$.

6) Nanda N, Barold SS, Gramiak R, Ong LS, Heinle RA. Echocardiographic features of right ventricular outflow tumor prolapsing into the pulmonary artery. Am J Cardiol 40: 272, 1977.

7) Noma S, Takahashi S, Sugie N, Nakajima H, Mitsuishi T, Akasaka T. Right ventricular myxoma of a young child originating from the tricuspid valve. Nihon-Shonikagakkai-Zasshi 93: 988, 1989 (abstract in English).

8) Chia BL, Lim C-H, Sheares JH, Choo MH. Echocardiographic findings in right ventricular myxoma. Am J Cardiol 58: 663, 1986. 